\title{
Childhood Ischemic Stroke: a Report of Two Cases
}

\author{
Uduma Felix Uduma (Corresponding author) \\ Department of Radiology, Abia State University Teaching Hospital \\ Aba, Nigeria \\ Tel: 234-803-745-0099Ｅ-mail: felixuduma@yahoo.com \\ Tadze Adamu Mbiydzela \\ Department of Radiology, Polyclinic Bonanjo \\ Douala, Cameroon \\ E-mail: mcd3ela@yahoo.com \\ Ngu Ernest \\ Department of Paediatrics, Polyclinic Bonanjo \\ Douala, Cameroon \\ Motah Mathieu \\ Neurosurgical unit, Department of Surgery, University of Douala \\ Douala, Cameroon \\ E-mail: motmath@yahoo.fr \\ Muna Wali \\ Department of Medicine, University of Yaounde \\ Yaounde, Cameroon \\ E-mail: walimuna@hotmail.com
}

Received: February 14, 2011 Accepted: March 15, 2011 doi:10.5539/gjhs.v3n2p200

\begin{abstract}
Rationale: Unlike in adults, stroke is uncommon in children. Even when it occurs, it is commonly haemorrhagic unlike ischemic predominance in adults. There is always a risk factor in childhood stroke. Aim: To present two case reports of childhood ischemic stroke managed conservatively. Case reports: case summary 1: A thirteen year old male Cameroonian Sickle with generalised body weakness, jaundice and right hemiparesis. Brain Computed tomogram (CT) showed premature generalised cerebral and cerebellar atrophy with supervening left parietal lobar ischaemic infarction. Bone window showed widened diploe. Abdomino-pelvic echogram revealed autosplenectomy and choleliathiasis. Case 2: A sixteen month old semi-conscious male Cameroonian with fever and left hemiplegia. Past medical history is Tetralogy of Fallot and Meningitis at 10 month of age. Brain Magnetic resonance imaging (MRI) showed right fronto-parietal cerebral venous infarct due to Superior sagital sinus thrombosis as evidenced by 'Empty delta sign'. Conclusion: Though stroke in children is uncommon more cases will be discovered with availability of neuro-imagings. The prognosis and prevention of stroke-repeat in Sicklers are better with blood transfusion.
\end{abstract}

Keywords: Stroke, Infarct, Ischemic

\section{Introduction}

The incidence of stroke in children is relatively low with 6 cases seen in every 100,000 children per year and one-third of these cases are seen in newborn [Children and stroke Online]. The occurrence of stroke in children 
has underlying risk factors which include Sickle cell disease (SCD) and thrombo-embolism [Chapman \&Nakiel 2003, pp 403-613]. Stroke in Sicklers is a relatively rare disorder with an incidence of 308/100,000[Ozawo et al Online]. Superior sagital sinus thrombosis (SSST) is an uncommon stroke and it is a diagnostic dilemma [Siegert et al, 1995, pp 496-7, Opatowsky et al, 1995, pp 414-7]. SSST constitutes about 72\% of aseptic cerebro-venous sinus thrombosis (CSVT) with incidence of CSVT in children being at least 0.67 per 100,000 per year [Clarkson, 2007, volume 10, number 2]

Aim: To present uncommon forms of childhood stroke with demonstration of radiological features.

\section{Case presentations}

\subsection{Case summary 1}

P.M (abbreviated name to conceal identity) is a 13year old male Cameroonian homozygous Sickler whose status was known before six months of life. He presented first in a hospital in Buea SW Region of Cameroon with clinical history of generalised body weakness, jaundice and previous convulsion. Physical examination revealed febrile, iteric small-for-age (weight 34kg), semi-conscious boy with left upward gaze. He was placed on anti-malarial drugs and vitamins. Laboratory investigations confirmed SS genotype with conjugated and unconjugated bilirubinaemia. He was then referred to a Neurosurgeon who requested for a cranio-cerebral computed tomography (CT) in Polyclinic Bonanjo, Douala. Serial axial slices of the brain were done using $2 \mathrm{~mm}$ from the base of the skull to the vertex with and without intravenous Iopamidol injections. Reconstructed images (coronal and sagital) and bone window were used in final image analysis. The CT findings showed generalised cerebral and cerebellar atrophy with non-enhancing peripherally based hypodense left parietal cerebral infarct. This is associated with left parieto-occipital lobar brain oedema. Bone window showed widening of the diploe and thinning of the inner table. The occipital bones are spared. Radiography of the hand showed medullary expansion with coarse trabeculae of the metacarpal and phalanges. Abdominal echograms showed mild hepatomegaly, autosplenomectomy, cardiomegaly and shadowing mobile gall stones. The largest diameter of the gall stone is $4.9 \mathrm{~mm}$. Patient was managed conservatively with blood transfusion, vitamins and other drugs with reasonable degree of neurological recovery.

\subsection{Case summary 2}

A 16 month old male Cameroonian baby with his name abbreviated to L.D. in order to preserve his identity was referred to Polyclinic Bonanjo, Douala for brain MRI. His presenting complaints were left hemiplegia, semi-consciousness and fever. Previous medical history is a diagnosis of Tetraology of Fallot and Meningitis at 10 months of life. Magnetic resonance images were acquired using 0.3Tesla AIRIS Hitachi machine. Magnetic resonance imaging (MRI) of the brain showed marked enlargement of the subarachnoid space on axial T1W (TR300, TE15) images. Sagital T1W (TR375, TE15) depicts the hyperintense content of the occipital subarachnoid space unlike the fronto-parietal region. This probably suggests some exsanguinations of variables ages. Axial T1W images show hyperintense superior sagital sinus and right transverse sinus. This hyperintensity of these sinuses are retained in T2W and FLAIR axial images. On contrast enhancement best shown on axial images, there is enhancement of both transverse sinuses and peripheral enhancement of superior sagital sinus with un-enhanced central lumen of the superior sagital sinus conforming to Empty Delta Sign. Axial FLAIR (TR7230, TE120) images show heterogenous right peri-insular gyri and right basal ganglia, these corresponding areas are enhanced and highlight a more extensive involvement of the entire right basal ganglia. The rest of the cerebral hemispheres show normal grey-white mater differentiations and normal myelination. Attempts at magnetic resonance venography were unsuccessful. A diagnosis of superior sagital sinus thrombosis with venous infarction of the right fronto-parietal cerebral lobes and right basal ganglia was made. Patient was managed conservatively after laboratory work-up with antibiotics and anti-coagulation with heparin.

\section{Discussion}

In paediatric series, Stroke has an underlying co-morbid conditions categorised into 3 patho-aetiologies viz:

(a) Thrombo-embolism-SCD, Congenital heart disease, Thalassaemia, Cardiomyopathy, Cerebrovenous thrombosis, Mitral valve prolapse, Atrial septal defect, Osler Weber Rendu syndrome, Systemic lupus erythromatosis, skull sepsis, MELAS (Myoclonal encephalopathy lactic acidosis syndrome).

(b) Haematological-Leukaemia, Thrombocytopaenia, Bleeding diasthesis

(c) Vasculopathies- Meningitis, Neurofibromatosis, Kawasaki’s disease, Polyarteritis nodosa, Extra-corporeal membrane oxygenations [Chapman \& Nakiely, 2003, pp403-613, Taylor, 1989, pp353-361, Carhuspoms et al., 1997, 2363-9]. 
Stroke is a catastrophic complications of SCD with reported risk of first stroke of 0.761 per 100 patients in their first 20years of life [Kwaku et al, 1998, pp288-294]. Unlike in adults were 79.2\% of strokes are thrombotic and 20.8\% are haemorrhagic [Onwuchekwa \& Onwuchekwa, 2009, pp25-31]. Stroke in children are commonly haemorrhagic because of coagulopathies like haemophilia A and B [Ozawo et al, Online]. But our 2 indexed patients had ischaemic strokes.

Though stroke is rare in SCD but 25\% of patients with SCD have neurological complications often caused by silent ischaemic changes leading to cerebral atrophy and reduced cognitive functions [Children and Stroke, Online,Chapman \& Nakiel,2003,pp403-613]. In SCD, vasculopathy resulting from intimal hypertrophy in combination with agglutination of rigid erythrocytes causes vessel occlusion and ischaemic infarction of the brain [Children and Stroke, Online, Chapman \& Nakiel, 2003, pp403-613]. Since this SCD is a systemic disease, the sickled red blood cells (RBC) occlude micro-vasculatures of both internal carotid and vertebro-basilar arterial systems of the brain leading to the diffuse cerebral and cerebellar atrophy seen in our patient.

The regional localisation of cerebral infarction in the parietal lobes conforms to Onwuchekwas assertion that parietal lobe is the second commonest site of infarction. This is because parietal lobe falls into the watershed vascular territory of the brain [Onwuchekwa\% Onwuchekwa, 2009, pp25-31]. Risk factors for stroke in SS children are transient ischaemic attack(TIA), meningitis, systolic BP, steady state leukocyte, acute chest syndrome within 2weeks before stroke and platelets counts [Kwaku et al, 1998,pp288-294].

There is a difference in stroke prevalence among SCD heterozygous variants and among ages [Kwaku et al, 1998, pp 288-294]. [Kwaku et al, 1998, pp 288-294]. Kwaku and Co-operative study of SCD et al found age adjusted prevalence estimate of $4.01 \%$ in SS patients, followed in decreasing order by the rates of $2.43 \%$ for S-B Thalassemia, $1.29 \%$ for S-B ${ }^{+}$Thalassemia and $0.84 \%$ for Haemoglobin SC. They also concluded that since children less than 2years of age had lower stroke incidence, there may be a protective mechanism operative in early life or the pathology responsible for stroke develops with time.

SSST is a subset of CSVT and CSVT is an unusual cause of stroke [Sebire, 2005, pp477-489]. The first case of SSST thereby bringing to cognizance CSVT was in the early part of $19^{\text {th }}$ Century, when Ribes in 1825 described the clinical and post-mortem spectrum of SSST. SSST could be septic or aseptic and 25\% idiopathic [Siegert et al, 1995, pp 496-497]. Infective causes are meningitis, brain abscess, sinusitis, mastoiditis, and trauma. Non-septic causes are haematological disorders, nephrotic syndrome, von Willebrand disease, Diabetes Mellitus, coagulopathies [Siegert et al, 1995, pp 496-497, Clarkson, 2007, volume 10, number 2, Sebire, 2005, pp 477-489].

CSVT is often unrecognised especially when posterior venous sinuses are affected therefore diagnosis is based on neuro-imaging like CT, MRI MRV with definitive diagnosis being arteriography [Opatowsky et al, 1995, pp414-417, Sigsbee et al, 1979.pp129-139]. The "empty delta sign" is a triangle of decreased density in the SSS on contrast enhanced computed tomography [Opatowsky et al. 1995, pp 414-417]. On MRI, it is a T1W and T2W hyperintense non-enhancing centre with enhancing hypointense T1W and T2W periphery in SSS[5].These CT and MRI findings are considered pathognomonic for SSST [Opatowsky et al.1995,pp 414-417]. The finding of such radiological sign is rare [Opatowsky et al.1995, pp 414-417]. CT may not be adequate in excluding this sign and must require contrast enhancement unlike MRI [Opatowsky et al.1995, pp 414-417].

This empty delta sign is a filling defect seen 1-4 weeks after onset of SSST [Shinohava et al, 1986, pp282-284]. The reliability of this sign is however questionable in extremes of acute and chronic stages of SSST due to complete occlusion or recanalisation respectively [Shinohava et al, 1986, pp282-284]. [Shinohava et al, 1986, pp 282-284]. Corroborating SSST in our patient is the presence of T1W and T2W high signal of right transverse sinus. This will present in $20 \%$ of cases as cord sign in non-contrast enhanced CT which present as alternating heterogenous hyperdense and hypodense areas in transverse sinus [Opatowsky et al.1995, pp 414-417]. Right transverse sinus is more affected in 75\% of cases than left one as seen in our patient [Taylor, 1989, pp 355-36]. MRI perfusion and diffusion weighted images play a role in detecting venous congestion and in differentiating cytotoxic and vasogenic oedema [Sabire, 2005, pp 477-489].

Management of stroke due to SSST IS based on systemic anticoagulation and anti-microbial whereas stroke due to SCD is based on blood transfusion [Magboo \& Lodhi, 2003, pp55-56]. [Magboo \& Lodhi, 2003, pp 55-56]. Magbool used blood transfusion every 6-8weeks in treating his SCD paediatric patient who had stroke but this patient had stroke relapse when she delayed her usual blood transfusion.

\section{Conclusion}

Though stroke in children is uncommon more cases will be discovered with availability of neuro-imagings. The prognosis and prevention of stroke -repeat in Sicklers are better with blood transfusion. 


\section{References}

Adams, R. J., Mckie, V. C., Hsu, L., et al. (1994). Prevention of the stroke by transfusion in children with Sickle Cell Anemia. Paediatr Radiol, 24, 92-95.

Carhuspoms, J. R., Mitsias, P., \& Levine, S. R. (1997). Cerebral venous thrombosis and anticardiolipin antibodies. Stroke, 28 (12), 2363-2369. http://dx.doi.org/10.1161/01.STR.28.12.2363

Chapman, S. \& Nakiel, R. (2003). Aids to Radiological differential diagnosis 4th ed. Saunders Edinburg, 403-613.

Clarkson, C. (2007). Aseptic Thrombosis of the Superior Sagital Sinus in Otherwise Healthy Female.

Beginning the Oral Contraceptive Pill. The Internet Journal of Emergency and Intensive Care Medicine, 10 (2).

Children and stroke, St. John's Hospital. [Online] Available: http://www.st-johns.org/services/stroke center/Children.aspx (Assessed on 21/1/2011).

Kasner, S. E. (2000), Stroke treatment-specific Considerations. Neuro Clin, 18 (2), 399-417.

Kwaku, O., Weiner, S. J., Sleeper, L. A., Miller, S. T., \& Embassy, S. (1998). Co-operative Study of Sickle Cell Disease et al., Cerebro-vascular Accidents in Sickle Cell Disease: Rates and Risk Factors. Blood, 91 (1), 288-294.

Magboo, A. \& Lodhi, A. M. (2003). Cerebrovascular accident in sickle cell disease. J Coll Physicians Surg Park, 13 (1), 55-56.

Onwuchekwa, R. C. \& Onwunchekwa, A. C. (2009). Computerised Tomography of the brain findings in Stroke patients in University of Port Harcourt. West African journal of Radiology, 16 (1), 25-31.

Opatowsky, M. J., Morris, P. P., Regan, J. D., \& Mewborne, J. D. et al (1995). Rapid Thromboectomy of Superior Sagital Sinus and Transverse Sinus Thrombosis with a Rheolytic Catheter Device. AJNR, (20), 414-417.

Ozawo, Y., Tugal, O., \& Kutscher, L. M. (2010). Approach to acute hemipariesis in children. [Online] Available: http://www.paediatricneurology.com/newpage21.html (Assessed on 20/7/2010).

Preul, M. C., Cendes, F., Just, N., \& Mohr, G. (1998). Intracranial aneurysms and Sickle cell anemia, Multiplicity and propensity for the Vertebro-basilar territory. Neurosurgery, 42, 971-977. http://dx.doi.org/10.1097/00006123-199805000-00007

Rononwden, S. (2004). Cerebral venous Sinus Thrombosis. Eur Radiol, 14, $215-216$. http://dx.doi.org/10.1007/s00330-003-2021-6

Safarn, B. A., Vighetto, A., Landins, T., \& Cabanis, E. (2004). Thrombosid veineuses Cerebrales In Neuro-opthalmologie Societe Francaise d'Opthalmologie. Pams, 498-502.

Sebire, G., Tabarki, B., Saundes, D. E., \& Leroy, I., et al. (2005). Cerebral Venous Sinus Thrombosis in Children: Risk Factors, Presentation, Diagnosis and Outcome. Brain, 128 (3), 477-489. http://dx.doi.org/10.1093/brain/awh412

Siegert, C. E. H, Smelt, A. H. M., \& de Bruin, T. W. A. (1995). Superior Sagital Sinus Thrombosis and Thyrotoxicosis, possible association in two cases. Stroke, (26), $496-497$. http://dx.doi.org/10.1161/01.STR.26.3.496

Sigsbee, B., Decl, M. D. F., \& Posner, J. B. (1979). Metastatic Superior Sagital Sinus Thrombosis Complicating Systemic Cancer. Neurology, 129-139.

Shinohava, Y., Yoshitoshu, M., \& Yoshi, F. (1986). Appearance of Delta Sign in Superior Sinus Thrombosis. Stroke, 17, 1282-1284. http://dx.doi.org/10.1161/01.STR.17.6.1282

Taylor, G. A., Fitz, C. R., Kapur, S., \& Short, B. L. (1989). Cerebrovascular accidents in neonates treated with extra-corporeal membrane oxygenation: Sonographic -pathological correlation. American Journal of Roentgenology, 153 (2), 355-361.

Vieira, J. P., Luis, C., Monteiro, J. P., Temudo, T., Campos, M. M., Quintes, S., \& Numes, J. (2010). Cerebral Sinovenous thrombosis in children: Clinical presentations and extension, localisation and recanalisation of thrombosis. European Journal of Paediatric Neurology, 14, 80-85. http://dx.doi.org/10.1016/j.ejpn.2008.12.004 


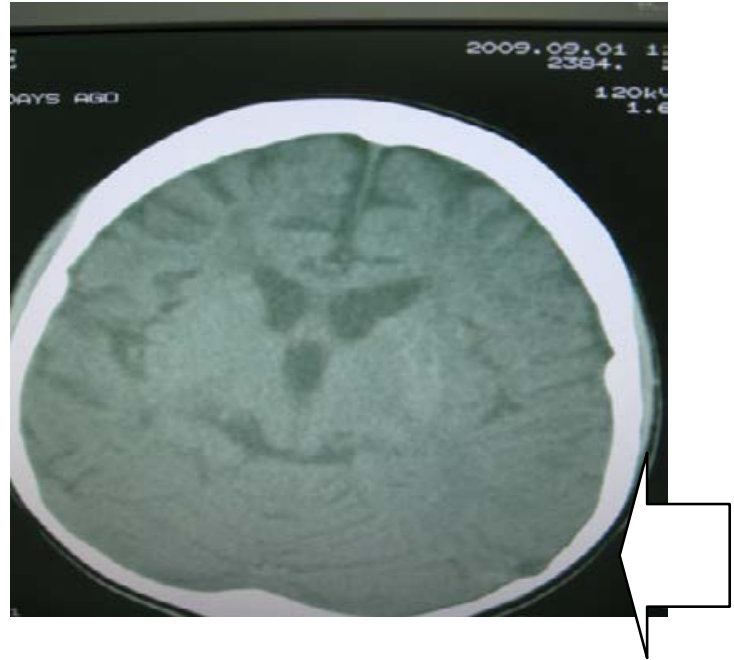

Figure 1. Axial CT showing diffuse cerebral \& cerebellar atrophy with smooth left parieto-occipital lobe

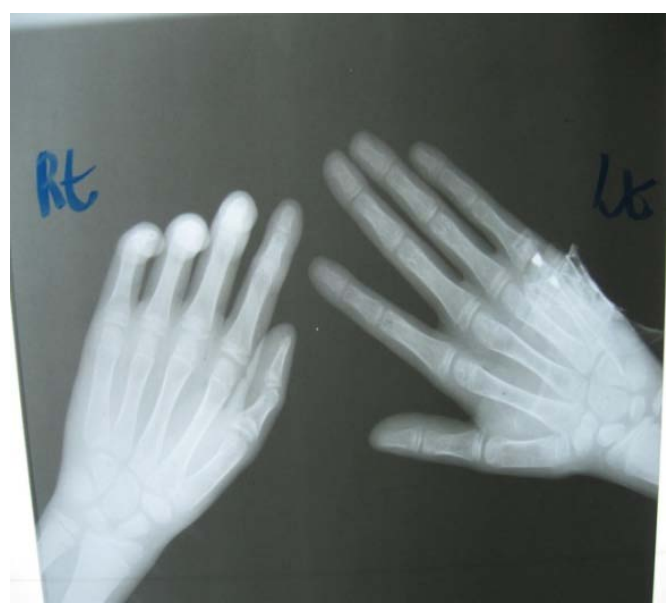

Figure 3. Hands radiograph showing medullary expansion

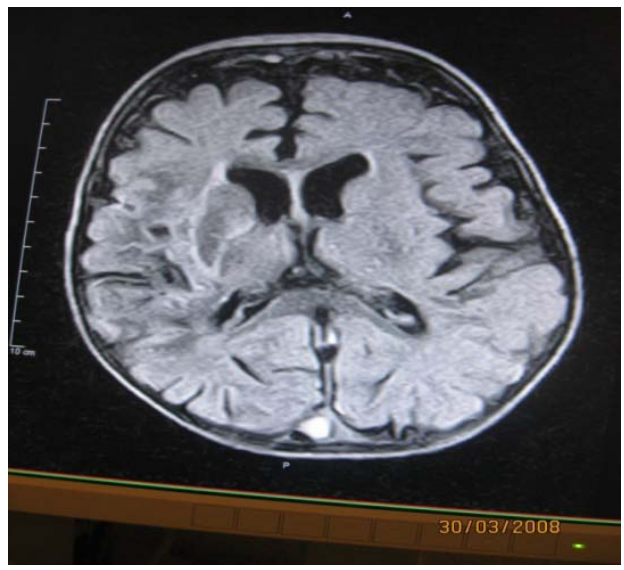

Figure 5. Axial T1W MRI showing hyperintense superior sagital sinus and patchy right fronto-parietal lobe

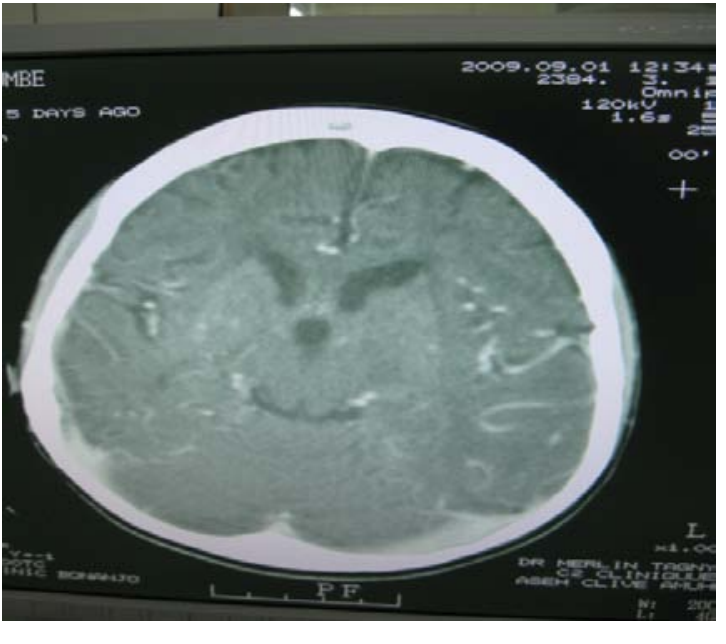

Figure 2. Enhanced axial CT showing some patchy gyral enhancement in left parieto-occipital lobe

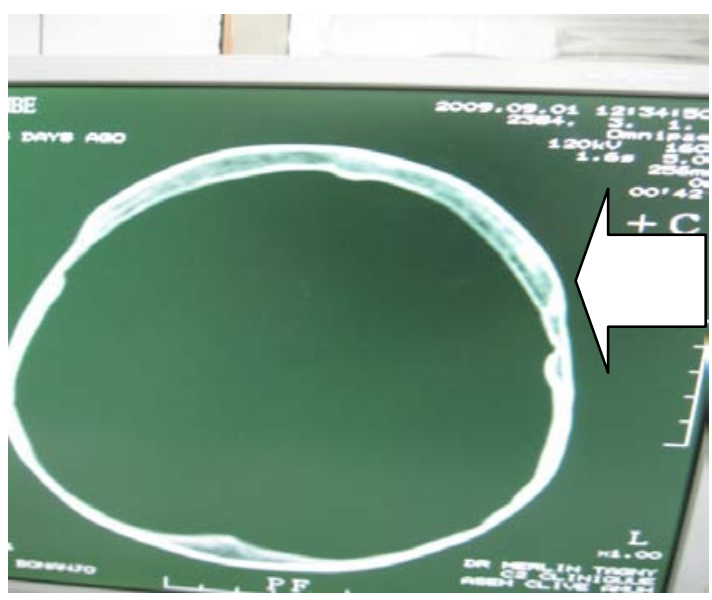

Figure 4. Skull bone window showing widened fronto-parietal diploe

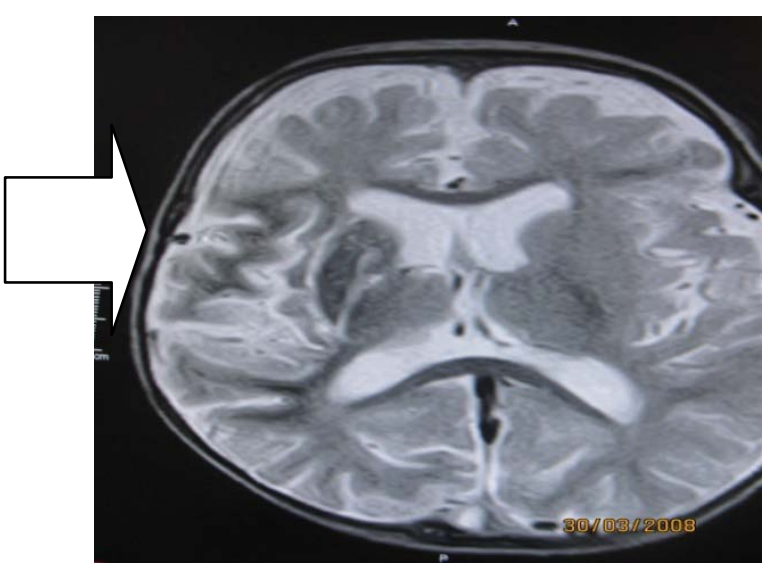

Figure 6. Axial T2W MRI showing hyperintense superior sagital sinus and patchy right fronto-parietal lobe 


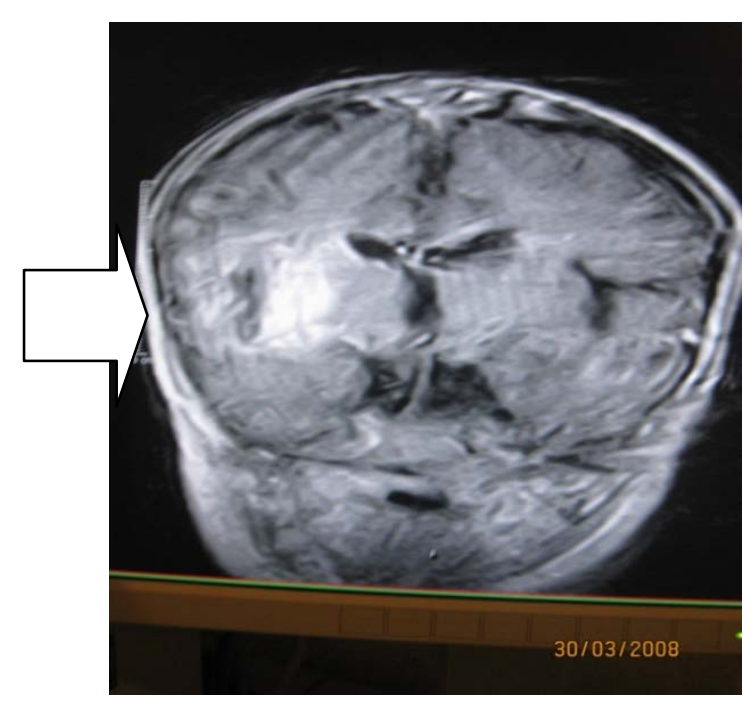

Figure 7. Coronal FLAIR MRI showing hyperintense deep white mater of right fronto-parietal lobe \& right basal ganglia

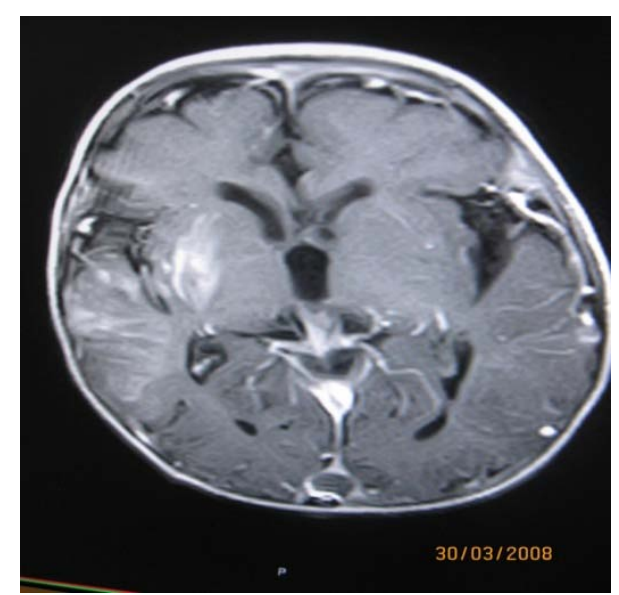

Figure 9. Axial TIW GD-DTPA MRI showing rim enhancement with unenhanced core of superior sagital sinus (EMPTY DELTA SIGN)

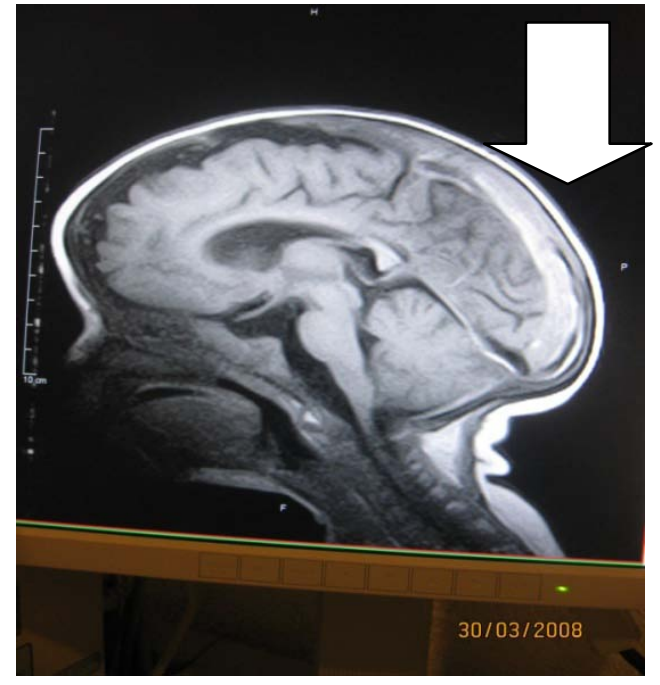

Figure 8. Sagital T1W MRI showing enlarged sub-arachnoid space with occipital part hyperintense

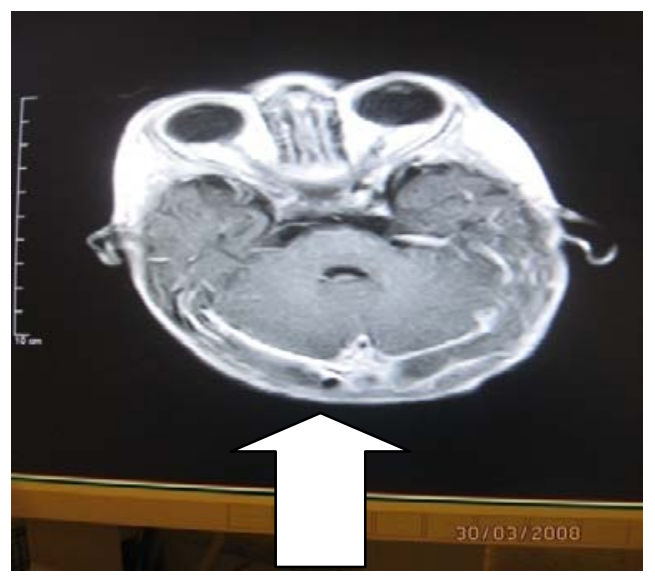

Figure 10. Axial GD-DTPA T1W MRI showing enlarged right transverse sinus 